ISSN 1997-5902

\title{
Influence de la salinité sur la structure des branchies et l'ultrastructure des ionocytes Chez le tilapia Sarotherodon melanotheron heudelotii provenant d'un estuaire hypersalé (Saloum, Sénégal).
}

\author{
N'Golo OUATTARA', Siaka OUATTARA'1, Yacouba BAMBA², Kouakou YAO1 \\ ${ }^{1}$ Laboratoire de Biologie et de Cytologie Animale, UFR-Sciences de la Nature, Université Nangui Abrogoua, 02 BP 801 \\ Abidjan 02, Côte d'Ivoire. \\ 'Laboratoire d'Environnement et de Biologie Aquatique (LEBA), UFR-Sciences et Gestion de l'Environnement, Université \\ Nangui Abrogoua, 02 BP 801 Abidjan 02 (Côte d'lvoire). \\ Auteur correspondant : N'Golo OUATTARA ouattarangolo_sn@una.edu.ci +225 45888899
}

Original submitted in on 10 April 2014. Published online at www.m.elewa.org on 31st July 2014. http://dx.doi.org/10.4314/jab.v79i1.5

\section{RESUME}

Objectif : Cette étude décrit la structure des branchies et les modifications ultrastructurales des ionocytes branchiaux du tilapia S. m. heudelotii provenant d'un estuaire hypersalé (Saloum, Sénégal) ; soumis à des variations de salinité de 0,$3 ; 35 ; 70$ et $90 \%$ au laboratoire durant $72 \mathrm{~h}$.

Méthodologie et résultats : Des juvéniles de $S$. $m$. heudelotii ont été soumis à des concentrations de salinité allant de l'eau douce à l'eau hypersalée. La structure des branchies et la localisation des ionocytes ont été étudiées par l'histologie photonique et l'ultratrastructure des ionocytes décrite par microscopie électronique à transmission. Quatre arcs branchiaux ont été identifiés. À $0,3 \%$, les ionocytes ont été observés sur les filaments, tandis qu'à $35 \%$ et plus, ils ont été localisés sur les filaments et les lamelles. Trois types d'ionocytes ont été identifiés selon la composition du cytoplasme et deux types en fonction de l'ouverture de la crypte apicale.

Conclusion et application : Cette étude montre pour la première fois que le tilapia S. m. Heudelotii s'adapte aux variations de concentration de salinité par la modification de la localisation et de l'ultrastructure de ses ionocytes branchiaux. Ces résultats suggérèrent que $S$. $m$. heudelotii peut également s'adapter parfaitement aux hypersalinités ce que d'autres espèces de tilapia ne supportent pas, en l'occurrence Oreochromis niloticus. II pourrait donc servir de modèle biologique dans les perspectives d'une pisciculture en eau saumâtre voire en eau de mer. En effet, l'élevage du tilapia se pratique principalement en eau douce qui est fortement concurrencée pour la consommation humaine et les usages industriels. Pourtant, les écosystèmes marins et lagunaires présentent d'énormes potentialités piscicoles mais très peu exploités. Par ailleurs, ces résultats pourraient aider à comprendre les phénomènes de spéciation chez certaines espèces liés aux perturbations environnementales provoquées par les changements climatiques.

Mots clés : Téléostéens, tilapia ; hypersalinité ; adaptation ; ionocytes ; ultrastructure. 

l'ultrastructure des ionocytes chez le tilapia provenant d'un estuaire hypersalé (Sénégal).

\section{ABSTRACT}

Objective: This study describes the structure of gills and the ultrastructural changes in gill ionocytes of the tilapia S. $m$. heudelotii from a hypersaline estuary (Saloum, Senegal), subject to variations in salinity of 0,$3 ; 35$; 70 and $90 \%$ in laboratory for $72 \mathrm{~h}$.

Methodology and Results: Juvenile S. m. heudelotii were subjected to concentrations of salinity ranging from freshwater $(0,3 \%)$ to hypersaline water $(90 \%)$. The gills structure and the location of ionocytes were studied by photonic histology and ionocytes ultratrastructure described by transmission electronic microscopy. Four gill arches were identified. At $0,3 \%$ of salinity, ionocytes were observed at the base of filaments, while at $35 \% 0$ and more, they have been examined on the filaments and lamellae. Three types of ionocytes were identified according to the composition of the cytoplasm and two types according to the opening of the apical crypt.

Conclusion and Application: This study shows for the first time that tilapia s. m. Heudelotii adapts to changes in concentration of salinity by modification of the location and ultrastructure of its gill ionocytes. These results suggested that this species can also fit perfectly into the high salinity, that other tilapia do not support, for example Oreochromis niloticus.

It might therefore serve as a biological model in the perspective of a fish farming in brackish water or seawater. Indeed, tilapia culture is carried out mainly in fresh water that is keenly competed for direct consumption by human people and for industrial uses. However, marine and lagoon ecosystems have enormous fish farming potential, but very less exploited. Furthermore, these results may help to understand the phenomena of speciation in some species associated with the environmental disruption caused by climate change.

Key words: Teleost; tilapia; hypersalinity; adaptation; ionocytes; ultrastructure

\section{INTRODUCTION}

En raison de l'importance et de l'insuffisance des eaux douces (ED) dans certaines régions du monde, les scientifiques se lance à la conquête des eaux saumâtres pour y produire des organismes d'eaux douces. Pour y parvenir, il est indispensable d'étudier la résistance à la salinité des organismes aquatiques dans leur contexte environnemental. $\mathrm{Ce}$ défi est particulièrement important pour la pisciculture des tilapias qui, malgré d'énormes potentiels aquacoles, restent essentiellement une production d'eau douce. Certaines espèces de tilapia telle que Sarotherodon melanotheron heudelotii dont les potentialités aquacoles ont pu être démontrées (Gilles et al., 1998) prospère dans les lagunes et estuaires côtiers d'Afrique de l'Ouest. Dans ces écosystèmes, les variations de salinité peuvent aller de $0,3 \%$ à $130 \%$. Ce tilapia pourrait être l'une des espèces les plus intéressantes pour être élevées dans de tels milieux. Chez la plupart des poissons, les fonctions osmorégulatrices sont assurées par des cellules spécialisées appelées ionocytes ou cellules riches en mitochondries ou encore cellules à chlorures (Marshall \& Bryson, 1998). Ce sont des cellules de grande taille (10-20 $\mu \mathrm{m}$ de hauteur), avec une ultrastructure caractéristique (Wilson \& Laurent, 2002). Une étude récente de Sardella et al. (2004) portée sur l'analyse de l'effet de l'hypersalinité sur la morphologie et l'ultrastructure des ionocytes branchiaux d'un tilapia hybride a révélé une variabilité marquée de la morphologie de la membrane apicale (Sardella et al., 2004). Ces résultats sont en accord avec ceux déjà rapportés par Franklin (1990) et Perry et al. (1992) chez d'autres espèces de poissons. Chez le tilapia $S$. melanotheron, seules quelques études ont décrit une régulation endocrinienne (Tine et al., 2007) et des modifications quantitatives des cellules à chlorures et de l'activité de la pompe $\mathrm{Na}^{+} / \mathrm{K}^{+}$-ATPase (Ouattara et al., 2009; Lorin-Nebel et al., 2012) en réponse à une augmentation de la salinité. Cependant, les modifications ultrastructurales des ionocytes de ce poisson ont été très peu étudiées. Le but de notre étude est de déterminer les caractéristiques morphologiques et ultrastructurales des ionocytes branchiaux liées à des variations de salinité chez $S$. m. heudelottii. 


\section{MATERIEL ET METHODES}

Site d'étude : L'estuaire du Sine Saloum est situé dans la zone soudano-sahélienne sur la façade atlantique de l'Afrique de l'ouest au Sénégal (Figure 1). II est compris entre $13^{\circ} 55^{\prime}$ et $14^{\circ} 10^{\prime}$ de latitude nord et $16^{\circ} 03^{\prime}$ et $16^{\circ} 50^{\prime}$ de longitude ouest (Diouf, 1996). Cet estuaire a été particulièrement affecté ces dernières années par des sécheresses consécutives; limitant fortement son approvisionnement en ED. Cette situation a engendré une inversion du fonctionnement de son gradient de salinité avec des concentrations plus fortes (130) en amont qu'en aval ( $40 \%)$ entrainant ainsi des situations de stress et une modification de structure (survie, distribution, métabolisme) chez les espèces de poisson qui peuplent cet écosystème

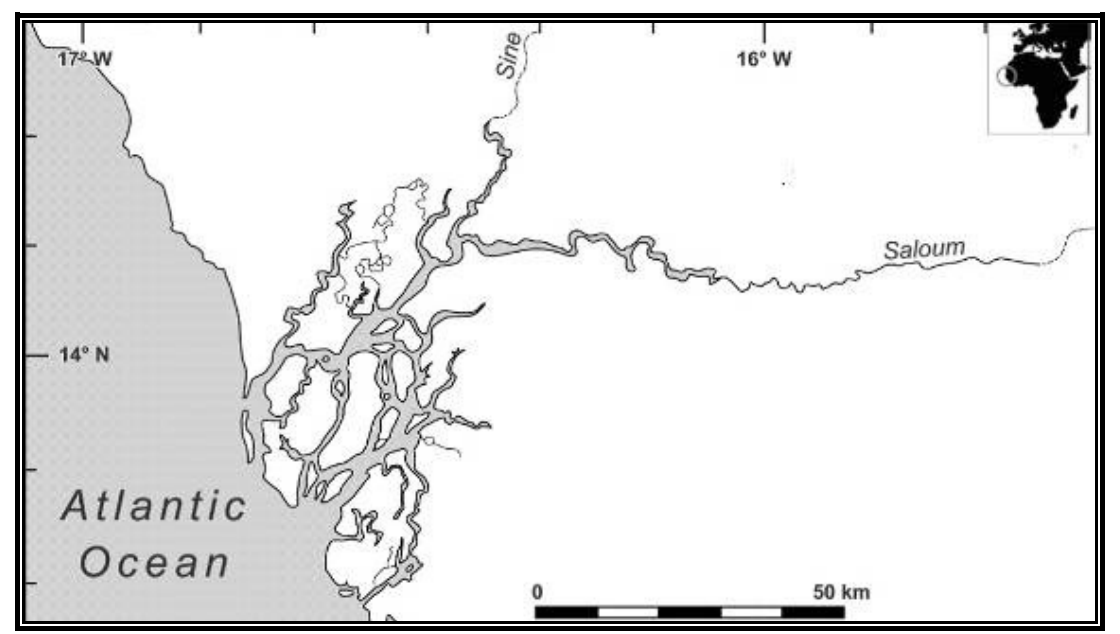

Figure 1 : Situation géographique de l'estuaire du Saloum (Sénégal).

Collecte des données : Des juvéniles de 35 jours d'âge $(0,25 \mathrm{~g}$ de poids moyen et $2,3 \mathrm{~cm}$ de longueur totale moyenne) issus de géniteurs collectés en août 2003 à Foundiougne au Sénégal dans l'estuaire du Saloum (salinité 48\%) et transportés à Montpellier en France, ont été utilisés pour cette étude. Ils ont été divisés en quatre lots de 40 poissons dans des aquariums de $37 \mathrm{~L}$ et maintenus à $27^{\circ} \mathrm{C}$ sous une photopériode naturelle. Un groupe a été maintenu dans l'ED $(0,3 \%)$ et a servi de lot témoin. Les trois autres groupes ont subi un transfert direct de l'ED à l'eau de mer (EM) $(35 \%)$ et une augmentation graduelle de $7 \%$ jour pour atteindre les salinités de 70 et $90 \%$. Trois individus par groupe ont été échantillonnés $72 \mathrm{~h}$ après le transfert aux différentes salinités. Après anesthésie des individus au 2-phénoxyéthanol $(2 \mathrm{ml} / \mathrm{l})$, les branchies ont été disséquées et sectionnées en petits morceaux. Les échantillons ont d'abord été préfixés dans un mélange à $4 \%(\mathrm{pH} 7,2)$, à volume égal d'une solution de glutaraldéhyde (350 mOsm. $\mathrm{kg}^{-1}$ ) et d'une solution tampon de cacodylate de sodium $0,1 \mathrm{M}$, pendant $24 \mathrm{~h}$ à température ambiante. $\mathrm{Ce}$ matériel biologique a ensuite été rincé 3 fois pendant une journée dans un tampon de cacodylate de sodium $0,2 \mathrm{M}$ (400 mOsm. $\left.\mathrm{kg}^{-1}\right)$ et fixé à nouveau dans une solution à volume égal de tétroxyde d'osmium à $2 \%(80 \mathrm{mOsm} . \mathrm{kg}$ 1) et du tampon cacodylate de sodium $0,1 \mathrm{M}$ (518 mOsm. $\mathrm{kg}^{-1}$ ), pendant $4 \mathrm{~h}$ à $4^{\circ} \mathrm{C}$. Les échantillons ont ensuite été rincés à l'eau distillée 3 fois /jour pendant 3 jours puis rapidement déshydratés dans des bains d'alcool éthylique de degrés croissants jusqu'à l'alcool absolu $\left(70,90,100^{\circ} \mathrm{C}\right)$ puis inclus dans l'EPON 812. La polymérisation de cette résine a été réalisée dans une étuve à $60^{\circ} \mathrm{C}$ pendant 3 jours. Des coupes semi-fines (1$1,5 \mu \mathrm{m})$ et ultrafines (60-90 nm) ont été réalisées à l'ultramicrotome LKB BROMMA 8800 ULTROTOME III, avec un couteau de diamant. Les coupes semi-fines obtenues ont été montées sur lame dans une goutte d'eau, puis séchées sur une platine chauffante et colorées au bleu de toluidine $(2,5 \mathrm{~g}$ de toluidine, $50 \mathrm{ml}$ de $\mathrm{Na} 2 \mathrm{CO} 3$ à $5 \%, \mathrm{pH}=11$ ). Après séchage à nouveau, le montage est récouvert d'une lamelle pour être observées au microscope photonique (Leica Diaplan) couplé à un appareil photographique numérique Leica DC $300 \mathrm{~F}$ associé au programme FW 4000. Les coupes ultrafines ont été contrastées à l'acétate d'uranyle et au citrate de plomb, et examinées au microscope électronique à transmission (MET) JEOL 1200 EX à 70 kV. 
Ouattara et al. J. Appl. Biosci. 2014. Influence de la salinité sur la structure des branchies et l'ultrastructure des ionocytes chez le tilapia provenant d'un estuaire hypersalé (Sénégal).

La microscopie photonique a été réalisée selon la technique de (Ouattara et al., 2009).

\section{RESULTATS}

Structure de la branchie de $S$. $m$. heudelotii : Les branchies de S. $m$. heudelotii ont une structure identique au schéma général observé chez les poissons téléostéens. Elles sont composées de 4 arcs branchiaux de chaque côté de la tête (Figure 2A). Les arcs sont composés de plusieurs filaments branchiaux qui portent de nombreuses lamelles (Figure 2B, C). Dans les espaces interlamellaires, les ionocytes sont disposés à partir de la base des lamelles en ED (Figure 2D) ; mais ils remontent en plusieurs couches le long des lamelles en eau hypersalée (EHS) (Figure 2E).

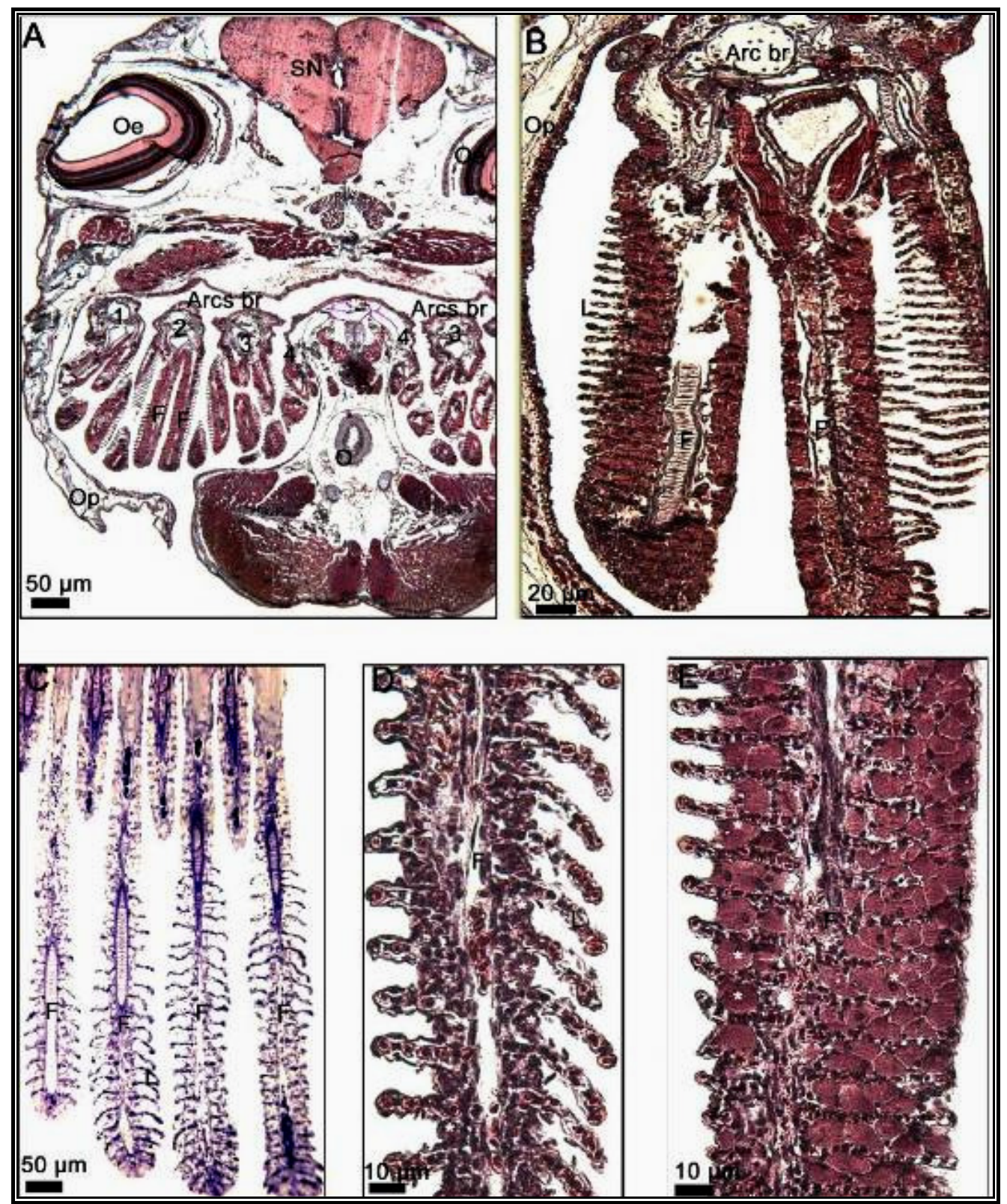

Figure 2 : Structure des branchies de Sarotherodon melanotheron et portions de filaments en ED et EHS. ED= eau douce, EHS = eau hypersalée. $S N=$ Système nerveux, Oe = OEil, O= OEsophage.

A: Organisation générale des 4 arcs branchiaux (Arcs br) de chaque côté des opercules (Op). 


\section{Ouattara et al. J. Appl. Biosci. 2014. Influence de la salinité sur la structure des branchies et l'ultrastructure des ionocytes chez le tilapia provenant d'un estuaire hypersalé (Sénégal).}

B: 1er arc branchial composé de plusieurs filaments $(F)$ portant des lamelles $(L)$. C: Filament $(F)$ portant les lamelles $(L)$ parallèles les unes aux autres et perpendiculaires aux filaments. D: Filament (F) en ED avec les ionocytes (étoiles) à la base des lamelles (L). E: Filament $(\mathrm{F})$ en EHS avec les ionocytes localisés aussi sur les filaments que sur les lamelles.

Ultrastructure des ionocytes: L'observation l'augmentation de la salinité (Figure 3B, C, D). En EM, les microscopique des coupes laisse apercevoir des modifications dans la structure branchiale en passant de I'ED à l'eau salée. En ED, les lamelles ont une forme régulière, fixées perpendiculairement au filament et parallèles les unes aux autres. Les ionocytes sont localisés dans les espaces interlamellaires à 15,56 $\mu \mathrm{m}$ de la base des lamelles (Figure $3 \mathrm{~A}$ ). Après le transfert en eau salée, l'extrémité des lamelles semble altérée avec lamelles sont quelque peu males formées et tuméfiées aux extrémités. Les ionocytes remontent la longueur des lamelles jusqu'à 22,22 $\mu \mathrm{m}$ de la base (Figure 3B). Cette distance augmente progressivement et atteint $26,67 \mu \mathrm{mà}$ 70 psu (Figure $3 \mathrm{C}$ ), puis environ $35,56 \mu \mathrm{m}$ à 90 psu (Figure 3D). Des cryptes apicales sont perceptibles sur certaines cellules en EHS (Figure 3C D).

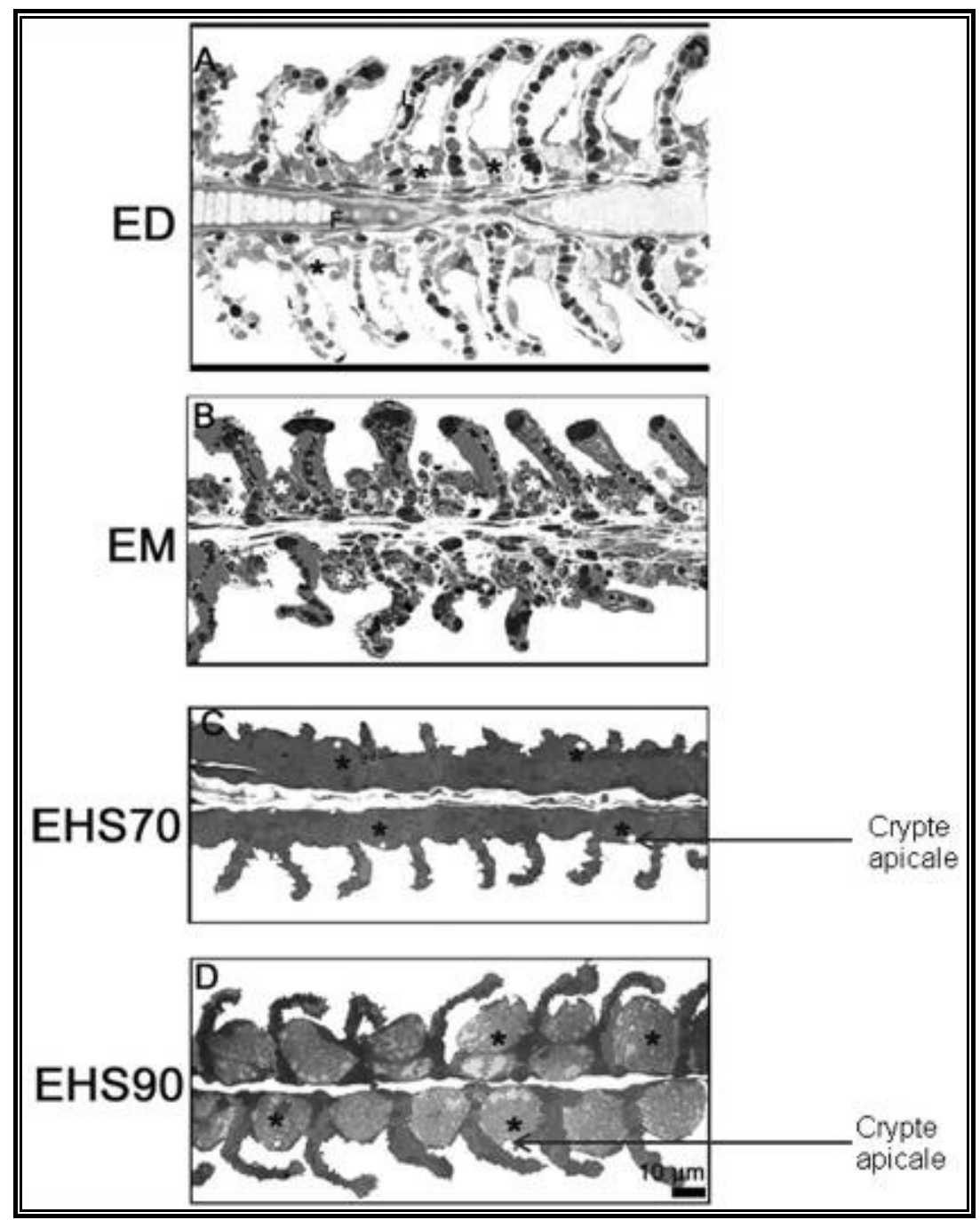

Figure 3 : Morphologie semi-fine des filaments $(F)$ et lamelles $(L)$ et localisation des ionocytes en fonction de la salinité chez S. m. heudelotii.

A: Filament en eau douce (ED). Noter la localisation des ionocytes (étoiles) sur le filament dans les espaces interlamellaires. B: Filament en eau de mer (EM). Noter l'hypertrophie des lamelles à la base et aux extrémités. C: Filament en eau hypersalée 70 psu (EHS70). Noter les grands ionocytes (étoiles) avec des cryptes apicales dans les espaces interlamellaires. D: Filament en eau hypersalée 90 psu (EHS90). Noter de très grands ionocytes (étoiles) souvent superposés dans les espaces interlamellaires avec des cryptes apicales. 

l'ultrastructure des ionocytes chez le tilapia provenant d'un estuaire hypersalé (Sénégal).

Les coupes ultrafines ont montré que l'épithélium de la lamelle branchiale est composée de deux couches distinctes séparées par un espace où peuvent circuler les érythrocytes (Figure 4A). La couche externe est formée de cellules pavimenteuses et ornée en surface de microcrêtes. La couche interne comprend des érythrocytes, des cellules respiratoires et des cellules piliers (Figure 4A). L'identification des cellules à chlores, basée sur les caractéristiques morphologiques du cytoplasme a permis de distinguer trois types d'ionocytes en ED : des ionocytes à cytoplasme clair avec les mitochondries clairsemées, des ionocytes à cytoplasme sombre avec des mitochondries plus groupés et des ionocytes immatures (Figure 4B, D). La forme des mitochondries varie de ronde ou ovoïde à allongée. Un vaste système tubulaire composé d'un réseau de tubules à paroi lisse (réticulum endoplasmique) connecté à la membrane basolatérale a également été observée (Figure 4E, F). Dans l'ensemble des cellules que nous avons dénommées cellules matures, ce réseau est étendu à l'ensemble du cytoplasme, à l'exception de l'étroite zone apicale, qui contient des vésicules subapicales clairement distinctes du système tubulaire (Figure 4B, D). Les noyaux de ces cellules sont clairs avec une chromatine peu développée (Figure 4B, D). Entre ces deux types cellulaires nous avons observé des cellules de petite taille avec une structure cellulaire incomplète composée souvent d'un cytoplasme contenant juste un noyau et parfois quelques mitochondries (Figure 4B, D)

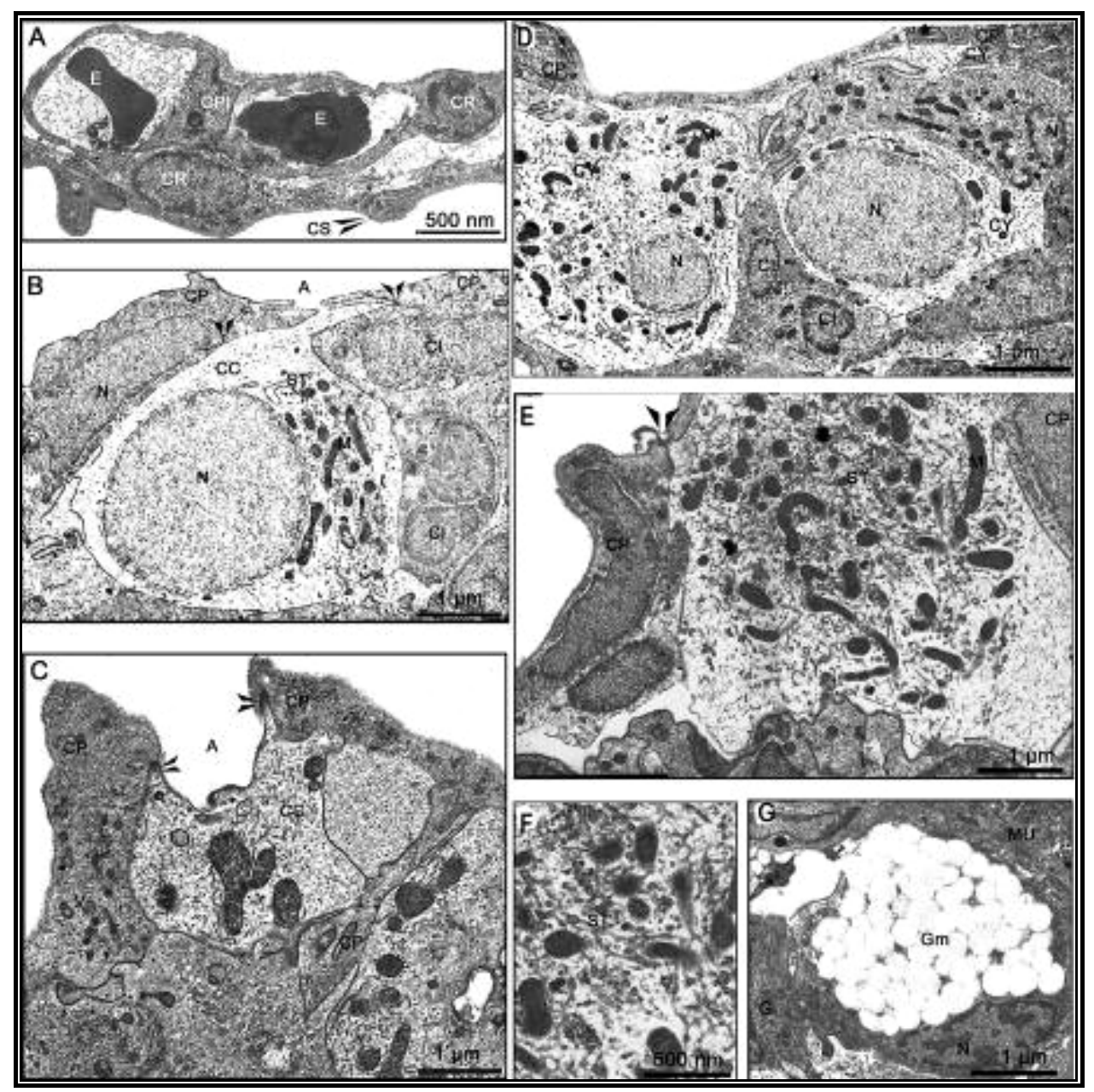

Figure 4: ultrastructure des ionocytes de S. m. heudelotii adapté en eau douce (ED).

A: Lamelle branchiale comprenant des érythrocytes $(\mathrm{E})$, des cellules piliers (CPI), des cellules respiratoires (CR) et des capillaires sanguins (CS). B, C, D, E et F: Complexe cellulaire (CC) formé de cellule à cytoplasme (CY) claire, à cytoplasme sombre et de cellules immatures (Cl). Noter la chromatine fine des noyaux (N), les cryptes apicales plates (A), les interdigitations (étoiles), un système tubulaire dense (ST), de nombreuses mitochondries (M), les cellules pavimenteuses (CP) et des jonctions serrées (flèche). 


\section{Ouattara et al. J. Appl. Biosci. 2014. Influence de la salinité sur la structure des branchies et l'ultrastructure des ionocytes chez le tilapia provenant d'un estuaire hypersalé (Sénégal).}

G: Mucocyte avec un cytoplasme ( $\mathrm{CY}$ ) contenant de nombreuses granules ( $\mathrm{Gr}$ ), un noyau (N) et des appareils de golgi (AG). Ces cellules sont dites immatures. L'ensemble de ces ionocytes (matures et immatures) sont entourés par un autre type cellulaire certainement les cellules pavimenteuses avec lesquelles les ionocytes forment des invaginations apicales (Figure 4B, D), et également de longues jonctions serrées qui partent souvent depuis la base pour se terminer dans la région apicale (Figure 4B, $\mathrm{C}, \mathrm{E})$. Ces cellules pavimenteuses ont une forme généralement polygonale (Figure $4 \mathrm{C}, \mathrm{E}$ ) avec un noyau allongé (Figure $4 \mathrm{E}$ ) et contiennent quelques mitochondries et un réseau dense de petites vésicules (Figure 4C). En fonction de l'ouverture des cryptes sur le milieu extérieur, deux types d'ionocytes ont été observés : des cellules à crypte apicale plate (Figure 4B) et d'autres à crypte plus en profondeur (Figure $4 \mathrm{C}$ ). De gros mucocytes ont été observés aussi bien en ED qu'en EM, caractérisés par de nombreuses granules à mucus et un système tubulaire avec des appareils de golgi (Figure $4 G)$.

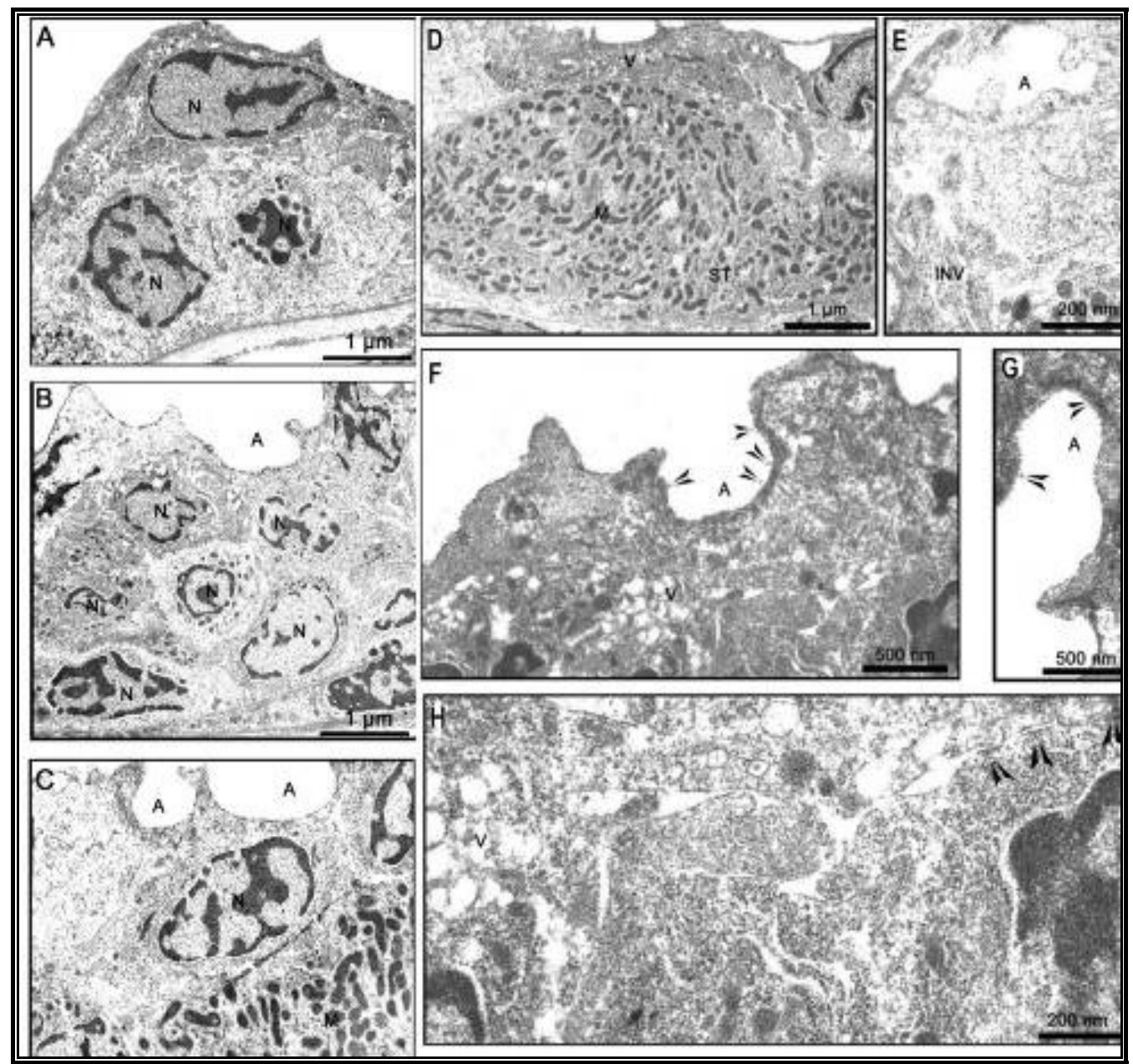

Figure 5: Différents types d'lonocytes dans les branchies de Sarotherodon melanotheron heudelotii adapté en eau de mer (EM).

A, B et C: Complexe cellulaire formé de cellules à cytoplasme claire et de cellules à cytoplasme sombre avec des cryptes apicales (A). Notez la dilatation de l'enveloppe nucléaire (N) et de nombreuses mitochondries (M) dans le cytoplasme. D: lonocyte avec de nombreuses mitochondries (M), un système tubulaire (ST) qui se dilate et les vésicules $(\mathrm{V})$ dans la région apicale. $E$ : Portion d'ionocyte montrant une crypte apicale (A), des invaginations (INV) et un système vésiculaire complètement éclaté. F: Portion d'ionocyte montrant le système tubulaire (V) éclaté, une crypte apicale avec un revêtement de glycoprotéines (flèches). G: Crypte apicale (A) avec revêtement de glycoprotéines (flèches). $\mathrm{H}$ : portion d'ionocyte montrant une jonction ouverte avec les desmosomes (flèches). 


\section{Ouattara et al. J. Appl. Biosci. 2014. Influence de la salinité sur la structure des branchies et l'ultrastructure des ionocytes chez le tilapia provenant d'un estuaire hypersalé (Sénégal).}

Les cellules à chlorure des animaux acclimatés en eau salée ont montré une nette différence du point de vu ultrastructurales avec celles des poissons en ED : les ionocytes sont groupés en un complexe multicellulaires formé d'ionocytes au cytoplasme claire et sombre (Figure $5 A, B)$ et la région apicale extrêmement vacuolisée (Figure 5D, F). Cependant, les limites entre les différentes cellules formant le complexe sont peu visibles. La surface apicale a été légèrement invaginée (Figure $5 E, H)$, le cytoplasme plus électro-dense (Figure 5D), la surface des noyaux quelque peu irrégulière avec une chromatine plus dense (Figure 5A, B, C), les mitochondries plus nombreuses, de forme allongée avec

\section{DISCUSSION}

La description et la quantification des cellules à chlorures fonctionnelles ont été menées chez de nombreuses espèces euryhalines telles que le tilapia Oreochromis mossambicus (Kültz et al., 1995, Lee et al., 2003), le tilapia hybride d'Oreochromis mossambicus $\mathrm{x}$ Oreochromis urolepis hornorum (Sarsella et al., 2004). Bien qu'il existe des différences dans l'anatomie générale des branchies entre les différents groupes de poissons, les cellules qui composent l'épithélium branchial sont très similaires (Wilson et Laurent, 2002). En utilisant la microscopie électronique à balayage, Lee et al. (1996) ont décrit pour la première fois chez des tilapias adaptés en ED, 3 types d'ionocytes en fonction des différences caractéristiques de la région apicale, partie apicale convexe et ondulée (type I), en forme de bassin (type II), crypte profonde (Type III). D'autre part, chez des tilapias adaptés à l'EM, un seul type de cellules à chlorures avec une crypte apicale profonde semblable à celle du type III en ED a été trouvé dans l'épithélium des branchies (Lee et al., 2000). Van der Heijden et al. (1997) ont également trouvé 3 types cellulaires chez le tilapia O. mossambicus adapté en ED et un seul type de cellule à chlorures dans les branchies de poissons élevés en EM. La présente étude basée sur la MET a démontré chez le tilapia Sarotherodon melanotheron adapté en ED, l'existence de deux types cellulaires en fonction de la forme de la crypte apicale, des cellules à crypte apicale plate que nous avons appelé type $A$ et des cellules à crypte apicale profonde nommées type $B$. Ce dernier type d'ionocytes pourrait correspondre au type III observé par Lee et al. (1996) et Van der Heijden et al. (1997, 1999) chez le tilapia O. mossambicus. La présence d'une crypte apicale a été décrite comme un élément distinctif des cellules à une électro-matrice dense et localisées en étroite association avec le système tubulaire (Figure $5 \mathrm{C}, \mathrm{F}, \mathrm{H}$ ). Ce système semble être hypertrophié, voir même désorganisé, formant un réseau dense de tubules anastomosées (Figure 5F, H). Des modifications majeures de l'ultrastructure telles que l'éclatement des vésicules dans le cytoplasme apical, qui semblent s'ouvrir à l'extérieur (Figure 5E, F, H) et la formation de jonctions intercellulaires ouvertes, caractérisées par un grand nombre de desmosomes (Figure $5 \mathrm{H}$ ) ont été observées. La membrane apicale en contact avec le milieu extérieur est recouverte par les glycoprotéines (Figure 5F, G).

chlorure des téléostéens marins (Laurent, 1984). Dans la présente étude, nous avons trouvé des cryptes apicales majoritairement peu profondes au niveau des ionocytes des poissons acclimatés en ED. Par contre, en EM les cryptes étaient bien profondes avec la membrane revêtue de glycoprotéines. Handy \& Eddy (1991) et Powell et al. (1994) ont indiqué que cette mucosubstance jouerait un rôle dans la concentration des cations dans le milieu intérieur et le maintien d'un gradient de concentration ionique. Le revêtement glycoprotéique observé sur la membrane apicale des ionocytes de $S$. melanotheron pourrait jouer ce même rôle. Les caractéristiques du cytoplasme nous ont permis également de distinguer 3 types d'ionocytes en ED, des cellules à cytoplasme clair et des cellules à cytoplasme sombre ainsi que des cellules de taille plus petite avec un cytoplasme parfois sans mitochondries que nous avons appelé cellules immatures ou indifférenciées. Même si les deux premiers types cellulaires semblent bien fonctionnels avec les mêmes composantes dans le cytoplasme, elles étaient formellement différentes par le contraste du cytoplasme. Des observations similaires basées sur la densité du cytoplasme ont permis à Wendelaar Bonga et al. (1990) de distinguer différents types d'ionocytes chez 0 . mossambicus. L'une des modifications ultrastructurales remarquables chez $S$. melanotheron a été la forte densité du système tubulaire basolatéral, lié à une augmentation de l'activité de la pompe $\mathrm{Na}^{+} / \mathrm{K}^{+}$-ATPase. Cette augmentation a été rapportée chez d'autres espèces de poisson (Uchida et al., 2000; Carmona et al., 2004). Les nombreuses mitochondries en association avec le système tubulaire contribueraient à produire l'énergie utilisée lors des échanges ioniques. 


\section{CONCLUSION}

Cette étude décrit les modifications ultrastructurales des ionocytes branchiaux du tilapia S. m. heudelotii soumis à des variations de salinité en milieu contrôlé, allant de l'ED à l'EHS en passant par l'EM. Une différence dans la localisation et les formes des ionocytes a été observée entre l'eau douce et l'eau salée. En ED, les ionocytes ont été observés à la base des filaments, tandis qu'en EM et

\section{REMERCIEMENTS}

Nous remercions particulièrement l'UMR Intrépid du Centre de coopération Internationale en Recherche Agronomique pour le Développement (CIRAD) de Montpellier et l'équipe Adaptation Ecophysiologique et Ontogenèse (AEO) UMR 5119 : ECOSYM de l'Université de Montpellier 2 pour le matériel biologique et l'expertise

\section{REFERENCES BIBLIOGRAPHIQUES}

Carmona R, García Gallego M, Sanz A, Domezaín A, Ostos Garrido MV, 2004. Chloride cells and pavement cells in gill epithelia of Acipenser naccarii: ultrastructural modifications in seawater acclimated specimens. Journal of Fish Biology 64: 553-566.

Diouf PS. 1996. Les peuplements de poissons des milieux estuariens de l'Afrique de l'Ouest : l'exemple de l'estuaire hypersalin du Sine Saloum. Thèse de doctorat, Universite Montpellier II : $267 \mathrm{pp}$.

Franklin GE, 1990. Surface ultrastructure changes in the gills of sockeye salmon (Teleostei: Oncorhynchus nerka) during seawater transfer: comparison of successful and unsuccessful seawater adaptation. Journal of Morphology 206: 13-23.

Gilles S, Amon-Kothias JB, Agnèse JF, 1998. In: JF Agnèse (Eds.), "Comparison of brackishwater growth performances of Sarotherodon melanotheron (Cichlidae) from three West African populations". Genetics and aquaculture in Africa: 199-210

Handy RD and Eddy FB, 1991. Effects of inorganic cation on $\mathrm{Na}+$ adsorption to the gill and body surface of rainbow trout, Oncorhynchus mykiss, in dilute solutions. Canadian Journal of Fisheries Aquatic Sciences 48: 1829-1837.

Kültz D, Jürss K, Jonas L, 1995. Cellular and epithelial adjustments to altered salinity in the gill and opercular epithelium of a cichlid fish (Oreochromis mossambicus). Cell and Tissue Research 279: 65-73. en EHS, ils ont été localisés sur les filaments et sur les lamelles. Deux types d'ionocytes ont été identifiés en fonction de l'ouverture de la crypte apicale et trois types selon la composition du cytoplasme. En EM et EHS les ionocytes ont formé des complexes multicellulaires et ont établie des jonctions laches avec les cellules pavimenteuses.

en microscopie électronique. Les auteurs sont reconnaissants au $\mathrm{Dr}$ Jean-François BAROILLER (directeur adjoint de l'UMR Intrépid) pour son soutien financier ce qui a rendu possible l'aboutissement de cette étude.

Laurent $P$, 1984. Gill internal morphology. In: Hoar WS, Randall DJ (Eds.), "Fish physiology", vol XA. Orlando, Academic Press: 73-183.

Lee TH, Feng SH, Lin CH, Hwang YH, Huang CL, Hwang PP, 2003. Ambient Salinity Modulates the Expression of Sodium Pumps in Branchial Mitochondria-Rich Cells of Mozambique Tilapia, Oreochromis mossambicus. Zoological Science 20: 29-36.

Lee TH, Hwang PP, Lin HC, Huang FL, 1996. Mitochondria-rich cells in the branchial epithelium of the teleosts, Oreochromis mossambicus, acclimated to various hypotonic environments. Fish Physiology and Biochemistry 15: 513-523.

Lee TH, Hwang PP, Shieh YE, Lin CH, 2000. The relationship between "deep-hole" mitochondriarich cells and salinity adaptation in the euryhaline teleost, Oreochromis mossambicus. Fish Physiology and Biochemistry 23: 133-140.

Lorin-Nebel C, Avarre J-C, Faivre N, Wallon S, Charmantier G, Durand J-D, 2012. Osmoregulatory strategies in natural populations of the black-chinned tilapia Sarotherodon melanotheron exposed to extreme salinities in West African estuaries. Journal of Comparative Physiology B DOI 10.1007/s00360-012-0657-8.

Marshall WS, Bryson SE, 1998. Transport mechaniss of seawater teleost chloride cells: An inclusive model of a multifunctional cell. Comparative Biochemistry and Physiology 119A: 97-106.

Ouattara N, Bodinier C, Nègre-Sadargues G, D'Cotta H, Messad S, Charmantier G, Panfili J, Baroiller J- 
$\mathrm{F}, 2009$. Changes in gill ionocyte morphology and function following transfer from fresh to hypersaline waters in the tilapia Sarotherodon melanotheron. Aquaculture 290: 155Ŕ164.

Perry SF, Goss GG, Fenwiwick JC, 1992. The interrelationship between gill chloride cell morphology and calcium uptake in freshwater teleost. Fish Physiology and Biochemistry 10: 327-337.

Powell MD, Speare DJ, Wright GM, 1994. Comparative ultrastructure morphology of lamellar epithelial, chloride and mucus cell glycocalyx of the rainbow trout (Oncorhynchus mykiss) gill. Journal of Fish Biology 44: 725-730.

Sardella BA, Matey V, Cooper J, Gonzalez RJ, Braunier CJ, 2004. Physiological, biochemical, and morphological indicators of osmoregulatory stress in "California" Mozambique tilapia (Oreochromis mossambicus $\times 0$. urolepis hornorum) exposed to hypersaline water. Journal of Experimental Biology 207: 13991413.

Tine M, Lorgeril J, Panfili J, Diop K, Bonhomme F, Durand JD, 2007. Growth hormone and Prolactin-1 gene transcription in natural populations of the black-chinned tilapia Sarotherodon melanotheron acclimatised to different salinities. Comparative Biochemistry and Physiology 147B: 541-549.

Uchida K, Kaneko T, Miyazaki H, Hasegawa S, Hirano T, 2000. Excellent salinity tolerance of Mozambique tilapia (Oreochromis mossambicus): Elevated chloride cell activity in the branchial epithelia of the fish adapted to concentrated seawater. Zoological Science 17: 149-160.

Van der Heijden AJH, Van der Meij JCA, Flik G, Wendelaar Bonga SE, 1999. Ultrastructure and distribution dynamics of chloride cells in tilapia larvae in freshwater and seawater. Cell and Tissue Research 297: 119-130.

Van der Heijden AJH, Verbost P, Eygensteyn J, Li J, Wendelaar Bonga SE, Flik G, 1997. Mitochondria-rich cells in gills of tilapia (Oreochromis mossambicus). Journal of Experimental Biology 200: 55-64.

Wendelaar Bonga SE, Flik G, Balm PHM, Van Der Meij JCA, 1990. The ultrastructure of chloride cells in the gills of teleost Oreochromis mossambicus during exposure to acidified water. Cell and Tissue Research 259: 575-585.

Wilson JM and Laurent $P, 2002$. Fish gill morphology: inside out. Journal of Experimental Zoology 293: 192-213. 